\title{
THE DADE GROUP OF A FUSION SYSTEM
}

\author{
MARKUS LINCKELMANN AND NADIA MAZZA
}

\begin{abstract}
We define a notion of a Dade group of a fusion system and show that some of the gluing and detection results for Dade groups of finite $p$-groups due to Bouc and Thévenaz in [8], [9] extend to Dade groups of fusion systems.
\end{abstract}

\section{INTRODUCTION}

The Dade group $D(P, \mathcal{F})$ of a fusion system $\mathcal{F}$ on a finite $p$-group $P$, for $p$ a prime, is defined as the subgroup of $\mathcal{F}$-stable elements in the Dade group $D(P)$ of $P$. The motivation for considering this notion comes from block theory: any choice of a maximal $b$-Brauer pair $(P, e)$ of a block $b$ of a finite group $G$ over an algebraically closed field $k$ of characteristic $p$ determines a fusion system $\mathcal{F}$ on $P$ - see e.g. [16] for details. Moreover, for any subgroup $Q$ of $P$ there is by [1] a unique block $e_{Q}$ of $k C_{G}(Q)$ such that $\left(Q, e_{Q}\right) \subseteq(P, e)$. Then, for any pair $(R, Q)$ of subgroups of $Q, R$ of $P$ such that $Q$ is normal in $R$ and such that the block $e_{Q}$ is a nilpotent block in the sense of [3], the unique (up to isomorphism) simple $k C_{G}(Q) e_{Q}$-module $V_{Q}$ becomes an endo-permutation $k R / Q$-module. In this way, any block gives rise to a fusion compatible family of endo-permutation modules for all sections $R / Q$ of $P$ for which the block $e_{Q}$ is nilpotent. One of the questions, first raised by Puig in [21], is whether this family can be "glued together" to an endo-permutation $k P$-module $V$ such that $\operatorname{Defres}_{R / Q}^{P}(V)$ is equal to $V_{Q}$ in the Dade group $D(R / Q)$ for any section $R / Q$ of $P$ as above. Following [21], this gluing problem has an affirmative answer if $P$ is abelian, which in turn is used to show the existence of a stable equivalence of Morita type between the block and its Brauer correspondent under the assumption that the inertial quotient acts freely on the non trivial elements of $P$. In [8], Bouc and Thévenaz gave a more general criterion for when the gluing problem has a solution provided that the involved endo-permutation modules are torsion in their respective Dade groups and $p$ is an odd prime. The purpose of the present paper is to investigate compatibility issues with respect to an arbitrary fusion system $\mathcal{F}$ on a finite $p$-group $P$. After a brief review on fusion systems in $\S 2$, we define in $\S 3$ the Dade group $D(P, \mathcal{F})$ of a fusion system $\mathcal{F}$ on a finite $p$-group $P$ and relate this to the definition of endo- $p$-permutation modules, due to Urfer [24]. In $\S 4$ and $\S 5$, we extend some results of Bouc and Thévenaz [8], [9] to this context, and in $\S 6$, we describe some examples of Dade groups of fusion systems.

\section{Generalities on FUSion Systems}

We review the definition of a fusion system, a concept due to Puig [19]. Our terminology follows [2] or [17].

Date: December 3, 2008.

Research of the second author was supported by Swiss National Research Fellowship PA002-113164/1. 
Definition 2.1. Let $P$ be a finite $p$-group. A fusion system on $P$ is a category $\mathcal{F}$ having as objects the subgroups of $P$; for any two subgroups $Q, R$ of $P$ the morphism set $\operatorname{Hom}_{\mathcal{F}}(Q, R)$ is a set of injective group homomorphisms with the following properties:

(1) composition of morphisms in $\mathcal{F}$ is the usual composition of group homomorphisms;

(2) if $\varphi: Q \rightarrow R$ is a morphism in $\mathcal{F}$ then so is the induced isomorphism $Q \cong \varphi(Q)$ as well as its inverse;

(3) $\operatorname{Hom}_{\mathcal{F}}(Q, R)$ contains the set $\operatorname{Hom}_{P}(Q, R)$ of group homomorphisms $\varphi: Q \rightarrow R$ for which there exists an element $y \in P$ satisfying $\varphi(u)=y u y^{-1}$ for all $u \in Q$;

(4) (I-S) if $\left|N_{P}(Q)\right| \geq\left|N_{P}(\varphi(Q))\right|$ for any $\varphi \in \operatorname{Hom}_{\mathcal{F}}(Q, P)$ then $\operatorname{Aut}_{P}(Q)$ is a Sylow $p$ subgroup of $\operatorname{Aut}_{\mathcal{F}}(Q)$;

(5) (II-S) if $\varphi: Q \rightarrow P$ is a morphism in $\mathcal{F}$ such that $\left|N_{P}(\varphi(Q))\right| \geq\left|N_{P}(\tau(Q))\right|$ for any $\tau \in \operatorname{Hom}_{\mathcal{F}}(Q, P)$ then $\varphi$ extends to a morphism $\psi: N_{\varphi} \rightarrow P$ in $\mathcal{F}$ where $N_{\varphi}$ is the subgroup of $N_{P}(Q)$ consisting of all $y \in N_{P}(Q)$ for which there exists $z \in N_{P}(\varphi(Q))$ with the property $\varphi\left(y u y^{-1}\right)=z \varphi(u) z^{-1}$ for all $u \in Q$.

Given a fusion system $\mathcal{F}$ on a finite $p$-group $P$, a subgroup $Q$ of $P$ is called fully $\mathcal{F}$-normalised if $\left|N_{P}(Q)\right| \geq\left|N_{P}(\varphi(Q))\right|$ for any $\varphi \in \operatorname{Hom}_{\mathcal{F}}(Q, P)$; similarly, $Q$ is called fully $\mathcal{F}$-centralised if $\left|C_{P}(Q)\right| \geq\left|C_{P}(\varphi(Q))\right|$ for any $\varphi \in \operatorname{Hom}_{\mathcal{F}}(Q, P)$. A subgroup $Q$ of $P$ is called $\mathcal{F}$-centric if $C_{P}(\varphi(Q))=Z(\varphi(Q))$ for any $\varphi \in \operatorname{Hom}_{\mathcal{F}}(Q, P)$, and $Q$ is called $\mathcal{F}$-radical if $\operatorname{Aut}_{Q}(Q)=$ $O_{p}\left(\operatorname{Aut}_{\mathcal{F}}(Q)\right)$, the largest normal $p$-subgroup of $\operatorname{Aut}_{\mathcal{F}}(Q)$. A subgroup $Q$ of $P$ is called $\mathcal{F}$-essential if $Q$ is $\mathcal{F}$-centric and if the partially ordered set of non-trivial $p$-subgroups of $\operatorname{Aut}_{\mathcal{F}}(Q) / \operatorname{Aut}_{Q}(Q)$ is not connected. If $Q$ is $\mathcal{F}$-essential then $Q$ is $\mathcal{F}$-centric radical. Alperin's fusion theorem implies that $\mathcal{F}$ is generated by the automorphism groups $\operatorname{Aut}_{\mathcal{F}}(Q)$ of $\mathcal{F}$-essential subgroups $Q$ of $P$. Following Stancu [23], the axioms (I-S) and (II-S) imply the a priori stronger axioms used in work of Broto-Levi-Oliver [2], where fusion systems are called saturated fusion systems.

Remark 2.2. Let $G$ be a finite group. For any two subgroups $Q, R$ of $G$ denote by $\operatorname{Hom}_{G}(Q, R)$ the set of injective group homomorphisms $\varphi: Q \rightarrow R$ for which there exists an element $x \in G$ satisfying $\varphi(u)=x u x^{-1}$ for all $u \in Q$. Let $p$ be a prime and let $P$ be a Sylow $p$-subgroup of $G$. The fusion system of $G$ on $P$ is the category denoted by $\mathcal{F}_{P}(G)$ having the subgroups of $P$ as objects and the sets $\operatorname{Hom}_{G}(Q, R)$ as morphism sets, for any two subgroups $Q, R$ of $G$. The category $\mathcal{F}_{P}(G)$ is a fusion system on $P$ in the sense of the definition 2.1. Axiom (3) of that definition implies that any fusion system $\mathcal{F}$ on $P$ contains the "trivial" fusion system $\mathcal{F}_{P}(P)$ of $P$ on itself.

Proposition 2.3. [2, Proposition 2.5] Let $\mathcal{F}$ be a fusion system on a finite p-group P. There is a finite $P$-P-biset $X$ with the following properties:

(1) Every transitive $P$ - $P$-subbiset of $X$ is of the form $P_{Q} \times{ }_{\varphi} P$ for some subgroup $Q$ of $P$ and some $\varphi \in \operatorname{Hom}_{\mathcal{F}}(Q, P)$.

(2) $\frac{|X|}{|P|}$ is congruent to 1 modulo $p$.

(3) For any subgroup $Q$ of $P$ and any $\varphi: Q \rightarrow P$ we have an isomorphism of $Q$-P-bisets ${ }_{\varphi} X \cong{ }_{Q} X$ and an isomorphism of $P$-Q-bisets $X_{\varphi} \cong X_{Q}$.

Here $P_{Q} \times{ }_{\varphi} P$ is the $P$-P-biset of equivalence classes in $P \times P$ with respect to the equivalence relation $(y u, z) \sim(y, \varphi(u) z)$, where $y, z \in P$ and $u \in Q$, and ${ }_{\varphi} X$ is the $Q$-P-biset equal to $X$ as 
right $P$-set with $u \in Q$ acting on $x \in X$, as $\varphi(u) x$; similarly for $X_{\varphi}$. Note that $P$ acts freely on the left and on the right of a biset $X$ as in Proposition 2.3.

\section{The Dade group of a fusion System}

Let $P$ be a finite $p$-group and let $k$ be a field of characteristic $p$. Following Dade [13], a $k P$ module $M$ is called an endo-permutation $k P$-module if $\operatorname{End}_{k}(M) \cong M \otimes_{k} M^{*}$ is a permutation $k P$-module. Here $M^{*}=\operatorname{Hom}_{k}(M, k)$ denotes the $k$-dual of $M$. Furthermore, $M$ is called an endotrivial $k P$-module if $\operatorname{End}_{k}(M) \cong k \oplus Y$ for some projective $k P$-module $Y$.

Given an endo-permutation $k P$-module $M$, its restriction $\operatorname{Res}_{Q}^{P}(M)$ to any subgroup $Q$ of $P$ has at most one isomorphism class of indecomposable direct summands with $Q$ as vertex. In particular, $M$ itself has at most one isomorphism class of indecomposable direct summands with $P$ as vertex, and any such summand is then called a cap of $M$. If $M$ has a cap we say that $M$ is capped. Note that $M$ is capped if and only if $\operatorname{End}_{k}(M)$ has a trivial direct summand as $k P$ module. In particular, any endotrivial $k P$-module is a capped endo-permutation $k P$-module. More precisely, an endotrivial $k P$-module restricts to any subgroup $Q$ of $P$ as the direct sum of one indecomposable endotrivial $k Q$-module and a projective $k Q$-module. If $M, N$ are capped endopermutation $k P$-modules then so is their tensor product $M \otimes_{k} N$. Two capped endo-permutation $k P$-modules $M, N$ are called equivalent if their caps are isomorphic. The Dade group of $P$ is the group of equivalence classes of capped endo-permutation $k P$-modules, with product induced by the tensor product over $k$. The inverse of the class of a capped endo-permutation $k P$-module $M$ in this group is the class of its $k$-dual $M^{*}$. The Dade group $D(P)$ is a finitely generated abelian group (cf. [20]). The equivalence classes whose cap is endotrivial form a subgroup of $D(P)$, denoted by $T(P)$. Note that two endotrivial $k P$-modules are equivalent if and only if they are isomorphic in the stable module category of $k P$-modules. The Dade group is contravariant functorial in $P$. If $P$ and $Q$ are two finite $p$-groups and $\varphi: Q \rightarrow P$ is a group homomorphism, we denote for any $k P$ module $M$ by $\operatorname{Res}_{\varphi}(M)$, or sometimes simply by ${ }_{v a r p h i} M$, the $k Q$-module which is equal to $M$ as $k$-vector space, with $u \in Q$ acting on $m \in M$ by $\varphi(u) m$. Restriction along $\varphi$ defines in this way an exact functor $\operatorname{Res}_{\varphi}: \bmod (k P) \rightarrow \bmod (k Q)$. If $M$ is a capped endo-permutation $k P$-module then $\operatorname{Res}_{\varphi}(M)$ is a capped endo-permutation $k Q$-module, and hence the functor $\operatorname{Res}_{\varphi}$ induces a group homomorphism between the corresponding Dade groups, still denoted by $\operatorname{Res}_{\varphi}: D(P) \rightarrow D(Q)$. This map sends $T(P)$ to $T(Q)$ and the torsion subgroup $D^{t}(P)$ of $D(P)$ to the torsion subgroup $D^{t}(Q)$ of $D(Q)$. If $\varphi$ is the inclusion morphism of a subgroup $Q$ of $P$ we write $\operatorname{Res}_{Q}^{P}$ instead of $\operatorname{Res}_{\varphi}$. We refer the reader to [7] for more background material and other usual notation concerning the Dade group. In particular, a section of a finite $p$-group $P$ is a pair $(T, S)$ of subgroups of $P$ such that $S \unlhd T \leq P$. If $R \leq S \unlhd T \leq P$, with $R \unlhd P$, we write

$$
\operatorname{Defres}_{T / S}^{P / R}=\operatorname{Def}_{T / S}^{T / R} \circ \operatorname{Res}_{T / R}^{P / R} \text { and } \quad \operatorname{Teninf}_{T / S}^{P / R}=\operatorname{Ten}_{T / R}^{P / R} \circ \operatorname{Inf}_{T / S}^{T / R} .
$$

As before, we use this notation for elements of the Dade group as well as for modules.

Definition 3.1. Let $P$ be a finite $p$-group and let $\mathcal{F}$ be a fusion system on $P$. The Dade group $D(P, \mathcal{F})$ of $(P, \mathcal{F})$ is the abelian group

$$
D(P, \mathcal{F})={\underset{\mathcal{L}}{\mathcal{F}}}_{\lim } \mathbf{D}
$$

where $\mathbf{D}$ denotes the contravariant functor from $\mathcal{F}$ to the category of abelian groups sending a subgroup $Q$ of $P$ to the Dade group $D(Q)$ and a morphism $\varphi: Q \rightarrow R$ in $\mathcal{F}$ to the group 
homomorphism $\operatorname{Res}_{\varphi}: D(R) \rightarrow D(Q)$. Similarly, we define

$$
\begin{aligned}
T(P, \mathcal{F}) & =\underbrace{\lim }_{\mathcal{F}} \mathbf{T}, \\
D^{t}(P, \mathcal{F}) & =\underbrace{\lim }_{\mathcal{F}} \mathbf{D}^{t},
\end{aligned}
$$

where $\mathbf{T}$ and $\mathbf{D}^{t}$ are the subfunctors of $\mathbf{D}$ sending a subgroup $Q$ of $P$ to $T(Q)$ and $D^{t}(Q)$, respectively.

Remark 3.2. We usually identify $D(P, \mathcal{F})$ with a subgroup of $D(P)$. With this identification, we have $T(P, \mathcal{F})=T(P) \cap D(P, \mathcal{F})$ and $D^{t}(P, \mathcal{F})=D^{t}(P) \cap D(P, \mathcal{F})$, which is the torsion subgroup of $D(P, \mathcal{F})$. Denoting as in 2.2 by $\mathcal{F}_{P}(P)$ the "trivial" fusion system of $P$ on itself we have obvious identifications $D(P)=D\left(P, \mathcal{F}_{P}(P)\right), T(P)=T\left(P, \mathcal{F}_{P}(P)\right)$, and $D^{t}(P)=D^{t}\left(P, \mathcal{F}_{P}(P)\right)$.

In order to describe $D(P, \mathcal{F})$ as subgroup of $D(P)$, we use the following terminology.

Definition 3.3. Let $P$ be a finite $p$-group and let $\mathcal{F}$ be a fusion system on $P$.

(1) A $k P$-module $M$ is called $\mathcal{F}$-stable if $\operatorname{Res}_{\varphi}(M) \cong \operatorname{Res}_{Q}^{P}(M)$ as $k Q$-modules for any subgroup $Q$ of $P$ and any $\varphi \in \operatorname{Hom}_{\mathcal{F}}(Q, P)$.

(2) An element $v \in D(P)$ is called $\mathcal{F}$-stable if $\operatorname{Res}_{\varphi}(v)=\operatorname{Res}_{Q}^{P}(v)$ in $D(Q)$, for any subgroup $Q$ of $P$ and any $\varphi \in \operatorname{Hom}_{\mathcal{F}}(Q, P)$.

In other words, the class $v$ in $D(P)$ of a capped endo-permutation $k P$-module $V$ is $\mathcal{F}$-stable if and only if the endo-permutation $k Q$-modules $\operatorname{Res}_{\varphi}(V)$ and $\operatorname{Res}_{Q}^{P}(V)$ have isomorphic caps, for any subgroup $Q$ of $P$ and any $\varphi \in \operatorname{Hom}_{\mathcal{F}}(Q, P)$. Thus the notion of $\mathcal{F}$-stability characterises elements in $D(P, \mathcal{F})$ :

Proposition 3.4. Let $P$ be a finite p-group and let $v \in D(P)$. Then $v \in D(P, \mathcal{F})$ if and only if $v$ is $\mathcal{F}$-stable.

Remark 3.5. Let $P$ be a finite $p$-group and let $\mathcal{F}$ be a fusion system on $P$. As a consequence of Alperin's fusion theorem, in order to determine whether a $k P$-module $M$ is $\mathcal{F}$-stable or not, it suffices to check that $\operatorname{Res}_{\varphi}(M) \cong \operatorname{Res}_{Q}^{P}(M)$ for every fully $\mathcal{F}$-normalised $\mathcal{F}$-essential subgroup $Q$ of $P$ and any $\varphi \in \operatorname{Aut}_{\mathcal{F}}(Q)$, viewed as morphism from $Q$ to $P$. Also, by property (4) of Definition 2.1, we can furthermore assume that $\varphi$ has order prime to $p$.

For endotrivial modules the notions of stability of a module and its class coincide:

Proposition 3.6. Let $P$ be a finite $p$-group. The class $v \in T(P)$ of an endotrivial $k P$-module $V$ is $\mathcal{F}$-stable if and only if $V$ is $\mathcal{F}$-stable.

Proof. This follows from the fact that for any subgroup $Q$ of $P$ we have $\operatorname{Res}_{Q}^{P}(V) \cong V_{Q} \oplus Y$, for some cap $V_{Q}$ of $\operatorname{Res}_{Q}^{P}(V)$ and some projective $k Q$-module $Y$.

For the class $v \in D(P)$ of an arbitrary capped endo-permutation $k P$-module $V$ it is still true that if $V$ is $\mathcal{F}$-stable then so is $v$. The converse, however, need not be true. However, by Green's Indecomposability Theorem, a capped endo-permutation $k P$-module $V$ can be written in the form

$$
V \cong \bigoplus_{R}\left(\operatorname{Ind}_{R}^{P}\left(V_{R}\right)\right)^{n_{R}},
$$


where $R$ runs over a set of representatives of the conjugacy classes of subgroups of $P$ and where $V_{R}$ is a cap of $\operatorname{Res}_{R}^{P}(V)$ for any subgroup $R$ of $P$, with uniquely determined integers $n_{R}$. The class of $V$ is $\mathcal{F}$-stable if $\operatorname{Res}_{\varphi}\left(V_{\varphi(R)}\right) \cong V_{R}$ for any morphism $\varphi: R \rightarrow P$ in $\mathcal{F}$. For the module $V$ itself to be $\mathcal{F}$-stable one would need further compatibility conditions on the integers $n_{R}$. What we show next is that any $\mathcal{F}$-stable class $v \in D(P)$ has at least some $\mathcal{F}$-stable representative.

Proposition 3.7. Let $\mathcal{F}$ be a fusion system on a finite p-group $P$ and let $v \in D(P, \mathcal{F})$. Then, there exists $V \in v$ such that $V$ is $\mathcal{F}$-stable.

Proof. Let $X$ be a $P$-P-biset with the properties as in Proposition 2.3. Consider the permutation $k P$ - $k P$-bimodule $k X$ with $k$-basis $X$. Let $v \in D(P, \mathcal{F})$ and let $W \in v$. We will show that the $k P$-module $V=k X \otimes_{k P} W$ is a capped endo-permutation $k P$-module belonging to $v$ and that $V$ is $\mathcal{F}$-stable. By property 2.3 (1) we have

$$
k X \cong \bigoplus_{(Q, \varphi)} k\left[P_{Q} \times{ }_{\varphi} P\right],
$$

with $(Q, \varphi)$ running over a suitable family of pairs consisting of a subgroup $Q$ of $P$ and a morphism $\varphi \in \operatorname{Hom}_{\mathcal{F}}(Q, P)$. By property $2.3(2)$ the bimodule $k X$ has a direct summand of the form ${ }_{\varphi} k P$ for some $\varphi \in \operatorname{Aut}_{\mathcal{F}}(P)$. Thus, by property $2.3(3)$ the bimodule $k X$ has in fact a direct summand isomorphic to $k P$ as $k P$ - $k P$-bimodule. Thus $W$ is isomorphic to a direct summand of $V$. The stability of $V$ follows from the stability property 2.3 (3) of the biset $X$. It remains to prove that $V$ is indeed an endo-permutation module. We have

$$
V \cong\left(\bigoplus_{(Q, \varphi)} k\left[P_{Q} \times{ }_{\varphi} P\right]\right) \otimes_{k P} W \cong \bigoplus_{(Q, \varphi)} k P \otimes_{k Q} \varphi^{k P} \otimes_{k P} W \cong \bigoplus_{(Q, \varphi)} \operatorname{Ind}_{Q}^{P}\left({ }_{\varphi} W\right)
$$

with $(Q, \varphi)$ as before. Since $v$ is $\mathcal{F}$-stable, each indecomposable summand of $V$ is isomorphic to $\operatorname{Ind}_{R}^{P}\left(W_{R}\right)$ for some subgroup $R$ of $P$, where $W_{R}$ is a cap of $\operatorname{Res}_{R}^{P}(W)$. Hence, to verify that $V$ is endo-permutation, it suffices to check that $\operatorname{Ind}_{R}^{P}\left(W_{R}\right) \otimes_{k} \operatorname{Ind}_{S}^{P}\left(W_{S}^{*}\right)$ is a permutation $k P$-module, for all subgroups $R, S$ of $P$. Now,

$$
\begin{gathered}
\operatorname{Ind}_{R}^{P}\left(W_{R}\right) \otimes_{k} \operatorname{Ind}_{S}^{P}\left(W_{S}^{*}\right) \cong \operatorname{Ind}_{R}^{P}\left(W_{R} \otimes \operatorname{Res}_{R}^{P}\left(\operatorname{Ind}_{S}^{P}\left(W_{S}^{*}\right)\right)\right) \cong \\
\quad \cong \operatorname{Ind}_{R}^{P}\left(\bigoplus_{x \in[R \backslash P / S]} W_{R} \otimes \operatorname{Ind}_{x_{S}}^{R}\left(\operatorname{Res}_{x_{S}{ }_{S}}^{x_{S}}\left(W_{x_{S}}^{*}\right)\right)\right) .
\end{gathered}
$$

Again by $\mathcal{F}$-stability of $W$, each indecomposable summand of $\operatorname{Ind}_{x_{S} \cap R}^{R}\left(\operatorname{Res}_{x_{S} \cap R}^{x_{S}}\left(W_{x_{S}}^{*}\right)\right)$ is isomorphic to $\operatorname{Ind}_{T}^{R}\left(W_{T}^{*}\right)$ for some subgroup $T \leq R$. Thus, the above is a direct sum of modules isomorphic to

$$
\operatorname{Ind}_{T}^{P}\left(\operatorname{Res}_{T}^{R}\left(W_{R}\right) \otimes_{k} W_{T}^{*}\right), \quad \text { for some subgroup } T \leq R .
$$

Now, each $\operatorname{Res}_{T}^{R}\left(W_{R}\right) \otimes_{k} W_{T}^{*}$ above is a direct summand of $\operatorname{Res}_{T}^{P}\left(W \otimes_{k} W^{*}\right)$, which is a permutation $k T$-module. Hence, so is $\operatorname{Res}_{T}^{R}\left(W_{R}\right) \otimes W_{T}^{*}$. Since a direct sum of permutation modules is still a permutation module, we conclude that $\operatorname{Ind}_{R}^{P}\left(W_{R}\right) \otimes_{k} \operatorname{Ind}_{S}^{P}\left(W_{S}^{*}\right)$ is a permutation $k P$-module, for all subgroups $R, S \in \mathcal{F}$. This shows that $V$ is an endo-permutation $k P$-module with the desired properties. 
We end this section with a comparison between $D(P, \mathcal{F})$ and the group $D_{P}(G)$, defined by Urfer in [24], when $P$ is a Sylow $p$-subgroup of $G$ and $\mathcal{F}=\mathcal{F}_{P}(G)$ is the fusion system defined by the action of $G$ on $P$ by conjugation. For sake of completeness, let us recall Urfer's definition.

Definition 3.8 (Définition 2.15, [24]). Let $G$ be a finite group and let $P$ be a Sylow $p$-subgroup of $G$.

(1) A $k G$-module $M$ is endo-p-permutation if $\operatorname{Res}_{P}^{G}(M)$ is endo-permutation. If so, then $M$ is capped if $\operatorname{Res}_{P}^{G}(M)$ is capped, i.e. has an indecomposable direct summand with vertex $P$.

(2) The set $D_{P}(G)$ of equivalence classes of capped endo-p-permutation $k G$-modules for the relation $M \sim N$ if $\left[\operatorname{Res}_{P}^{G} M\right]=\left[\operatorname{Res}_{P}^{G} N\right]$ in $D(P)$ is an abelian group. In particular, $D_{P}(P)=D(P)$.

Note that the equivalence relation identifies the endo- $p$-permutation modules whose caps have isomorphic sources. In Proposition 2.10 of [24], Urfer shows that an indecomposable endo-permutation $k P$-module $V$ has the property that $\operatorname{Ind}_{P}^{G}(V)$ is an endo- $p$-permutation $k G$-module if and only if $V$ is $G$-stable (that is, $\operatorname{Res}_{x P \cap P}^{x_{P}}\left({ }^{x} V\right) \cong \operatorname{Res}_{x P \cap P}^{P}(V)$, for all $\left.x \in G\right)$. From this, the author deduces an isomorphism between the subgroup of $G$-stable elements in $D(P)$ and the group $D_{P}(G)$. Translating the $G$-stability in terms of $\mathcal{F}$-stability, with $\mathcal{F}=\mathcal{F}_{P}(G)$, his result becomes:

Proposition 3.9 (Propositions 2.10 and 2.19, [24]). Let $G$ be a finite group and let $P$ be a Sylow $p$-subgroup of $G$. The restriction functor $\operatorname{Res}_{Q}^{G}: \bmod (k G) \rightarrow \bmod (k P)$ induces an isomorphism of abelian groups $\operatorname{Res}_{P}^{G}: D_{P}(G) \rightarrow D(P, \mathcal{F})$, with inverse induced by the induction functor $\operatorname{Ind}_{P}^{G}$ applied to $\mathcal{F}$-stable modules.

Note that we reach the same conclusion by applying Proposition 3.7.

Remark 3.10. Let us point out the following two observations.

(1) Since two capped endo- $p$-permutation modules are equivalent if and only if their caps have isomorphic sources, we have that $0 \in D_{P}(G)$ is the set of all $p$-permutation modules whose cap is a Scott module (i.e. isomorphic to a direct summand of the permutation module $k[G / P])$.

(2) The definition of the group $T(G)$ of endotrivial $k G$-modules, as given in [10], does not identify $T(G)$ to a subgroup of $D_{P}(G)$ unless $P=G$. Indeed, in $T(G)$, the elements are isomorphism classes of modules in the stable module category. Hence, two indecomposable endotrivial modules having isomorphic sources are generally not equivalent. Consequently, the kernel of the restriction map $\operatorname{Res}_{P}^{G}: T(G) \rightarrow T(P, \mathcal{F})$ is a finite group, isomorphic to the group generated by the isomorphism classes of the Scott modules that are endotrivial (i.e. such that $\operatorname{Res}_{P}^{G}(M) \cong k \oplus L$ for some projective $k P$-module $L$, cf. $[10, \S 2]$ ). Besides this fact, let us point out that it is yet still an open question to determine whether the map $\operatorname{Res}_{P}^{G}$ above is surjective or not. In other words, since for an (indecomposable) endo- $p$ permutation $k G$-module $M$ such that $\left[\operatorname{Res}_{P}^{G}(M)\right] \in T(P)$ does not guarantee that $\operatorname{Res}_{P}^{G}(M)$ is a 'genuine' endotrivial module, the conclusion is that $M$ has an endotrivial source, but is a priori not endotrivial (however, $M$ is the Green correspondent of an endotrivial $k N_{G}(P)$ module). 


\section{Detection}

"Detection" type results describe families $\mathcal{C}_{\mathcal{Y}}$ of sections $(T, S)$ of a finite $p$-group $P$ for which the product of the maps $\operatorname{Defres}_{T / S}^{P}: D(P) \rightarrow D(T / S)$ yields an injective map

$$
\operatorname{Defres}_{\mathcal{C}_{\mathcal{Y}}}: D(P) \longrightarrow \prod_{(T, S) \in \mathcal{C}_{\mathcal{Y}}} D(T / S)
$$

Definition 4.1. Let $\mathcal{Y}$ be a class of finite $p$-groups closed under taking sections and isomorphisms. Let $P$ be a finite $p$-group, let $\mathcal{F}$ be a fusion system on $P$ and let $\mathcal{C}$ be a subcategory of $\mathcal{F}$ closed under isomorphisms. We denote by $\mathcal{C}_{\mathcal{Y}}$ the category defined as follows:

- the objects of $\mathcal{C}_{\mathcal{Y}}$ are the sections $(T, S)$ of $P$ belonging to the subcategory $\mathcal{C}$ of $\mathcal{F}$ such that the quotient group $T / S$ belongs to $\mathcal{Y}$.

- the morphism set $\operatorname{Hom}_{\mathcal{C}_{\mathcal{Y}}}\left((T, S),\left(T^{\prime}, S^{\prime}\right)\right)$ is the set of morphisms $\varphi: T \rightarrow T^{\prime}$ in $\mathcal{C}$, such that $S^{\prime} \leq \varphi(S)$, for any two objects $(T, S),\left(T^{\prime}, S^{\prime}\right)$ in $\mathcal{C}_{\mathcal{Y}}$, with composition induced by that in $\mathcal{F}$. If $\mathcal{C}=\mathcal{F}_{P}(P)$ we write $P_{\mathcal{Y}}$ instead of $\mathcal{F}_{P}(P) \mathcal{Y}$.

With the notation above, given a morphism $\varphi:(T, S) \rightarrow\left(T^{\prime}, S^{\prime}\right)$ in $\mathcal{C}_{\mathcal{Y}}$, the groups $\varphi(T), \varphi(S)$ belong to $\mathcal{C}$ because $\mathcal{C}$ is assumed to be closed under isomorphisms, and the section $(\varphi(T), \varphi(S))$ of $P$ can be identified with the section $\left(\varphi(T) / S^{\prime}, \varphi(S) / S^{\prime}\right)$ of $T^{\prime} / S^{\prime}$. Hence the map $\operatorname{Defres}_{\varphi(T) / \varphi(S)}^{T^{\prime} / S^{\prime}}$ : $D\left(T^{\prime} / S^{\prime}\right) \rightarrow D(\varphi(T) / \varphi(S))$ is well-defined. Restriction along the isomorphism $T / S \cong \varphi(T) / \varphi(S)$ induces an isomorphism $D(\varphi(T) / \varphi(S)) \cong D(T / S)$ which we will denote abusively by $\operatorname{Res}_{\varphi}$ again. In this way taking Dade groups of the quotients $T / S$ yields a contravariant functor on $\mathcal{C}_{\mathcal{Y}}$.

Definition 4.2. Let $P$ be a finite $p$-group, let $\mathcal{F}$ be a fusion system on $P$, let $\mathcal{C}$ be a subcategory of $\mathcal{F}$ closed under isomorphisms, and let $\mathcal{Y}$ be a class of finite $p$-groups closed under taking sections and isomorphisms. Denote by $\mathbf{D}: \mathcal{C}_{\mathcal{Y}} \rightarrow \mathcal{A}$ the contravariant functor sending an object $(T, S)$ in $\mathcal{C}_{\mathcal{Y}}$ to the group $D(T / S)$ and a morphism $\varphi:(T, S) \rightarrow\left(T^{\prime}, S^{\prime}\right)$ in $\mathcal{C}_{\mathcal{Y}}$ to the group homomorphism $\operatorname{Defres}_{\varphi}=\operatorname{Res}_{\varphi} \circ \operatorname{Defres}_{\varphi(T) / \varphi(S)}^{T^{\prime} / S^{\prime}}: D\left(T^{\prime} / S^{\prime}\right) \rightarrow D(T / S)$. We set

$$
\underset{\leftarrow}{\mathbf{D}}\left(P, \mathcal{C}_{\mathcal{Y}}\right)=\lim _{\mathcal{C}_{\mathcal{Y}}}(\mathbf{D})
$$

Similarly, we set $\underset{\leftarrow}{\mathbf{D}^{t}}\left(P, \mathcal{C}_{\mathcal{Y}}\right)=\underset{\overleftarrow{\mathcal{C}_{\mathcal{Y}}}}{\lim }\left(\mathbf{D}^{t}\right)$ and $\underset{\leftarrow}{\mathbf{T}}\left(P, \mathcal{C}_{\mathcal{Y}}\right)=\underset{\overleftarrow{\mathcal{C}_{\mathcal{Y}}}}{\lim }(\mathbf{T})$, where $\mathbf{D}^{t}$ and $\mathbf{T}$ are the subfunctors of $\mathbf{D}$ sending an object $(T, S)$ in $\mathcal{C}_{\mathcal{Y}}$ to the subgroups $D^{t}(T / S)$ and $T(T / S)$ of $D(T / S)$, respectively. We denote by

$$
\operatorname{Defres}_{(\mathcal{C}, \mathcal{Y})}^{\mathcal{F}}: D(P, \mathcal{F}) \rightarrow \underset{\leftarrow}{\mathbf{D}}\left(P, \mathcal{C}_{\mathcal{Y}}\right)
$$

the canonical map determined by the family of maps $\operatorname{Defres}_{T / S}^{P}: D(P, \mathcal{F}) \rightarrow D(T / S)$, where $(T, S)$ runs over the objects of $\mathcal{C}_{\mathcal{Y}}$. If $\mathcal{C}=\mathcal{F}_{P}(P)$ we write $\underset{\leftarrow}{\mathbf{D}}\left(P_{\mathcal{Y}}\right)$ instead of $\underset{\leftarrow}{\mathbf{D}}\left(P, \mathcal{F}_{P}(P)_{\mathcal{Y}}\right)$ and $\operatorname{Defres}_{\mathcal{Y}}: D(P) \rightarrow D\left(P_{\mathcal{Y}}\right)$ instead of $\operatorname{Defres}_{\left(\mathcal{F}_{P}(P), \mathcal{Y}\right)}^{\mathcal{\mathcal { F }}} \cdot$

Remark 4.3. We can identify the group $\mathbf{D}\left(P, \mathcal{C}_{\mathcal{Y}}\right)$ with the set of families $\left(v_{(T, S)}\right)_{(T, S) \in \mathcal{C}_{\mathcal{Y}}}$ with $v_{(T, S)} \in D(T / S)$ such that for any morphism $\varphi:(T, S) \rightarrow\left(T^{\prime}, S^{\prime}\right)$ in $\mathcal{C}_{\mathcal{Y}}$, we have that

$$
\operatorname{Defres}_{\varphi}\left(v_{\left(T^{\prime}, S^{\prime}\right)}\right)=v_{(T, S)} \cdot
$$


With this identification, the groups $\mathbf{D}^{t}\left(P, \mathcal{C}_{\mathcal{Y}}\right), \mathbf{T}\left(P, \mathcal{C}_{\mathcal{Y}}\right)$ become subgroups of $\mathbf{D}\left(P, \mathcal{C}_{\mathcal{Y}}\right)$, the group $\mathbf{D}(P, \mathcal{F} \mathcal{Y})$ becomes a subgroup of $\left.\underset{\mathbf{D}}{\leftarrow} P_{\mathcal{Y}}\right)$. More generally, if $Q$ is a subgroup of $P$ and $\mathcal{G}$ a fusion system on $Q$ such that $\mathcal{G} \subseteq \mathcal{F}$, then $\mathcal{G}_{\mathcal{Y}}$ is a subcategory of $\mathcal{F}_{\mathcal{Y}}$, and the inclusion functor $\mathcal{G}_{\mathcal{Y}} \subseteq \mathcal{F}_{\mathcal{Y}}$ induces a canonical group homomorphism

$$
\underset{\leftarrow}{\mathbf{D}}\left(P, \mathcal{F}_{\mathcal{Y}}\right) \rightarrow \underset{\leftarrow}{\mathbf{D}}\left(Q, \mathcal{G}_{\mathcal{Y}}\right),
$$

mapping $\left(v_{(T, S)}\right)_{(T, S) \in \mathcal{F}_{\mathcal{Y}}}$ to its canonical projection $\left(v_{(T, S)}\right)_{(T, S) \in \mathcal{G}_{\mathcal{Y}}}$. In particular, we have a canonical map $\underset{\leftarrow}{\mathbf{D}}(P \mathcal{Y}) \rightarrow \underset{\leftarrow}{\mathbf{D}}\left(Q_{\mathcal{Y}}\right)$.

Proposition 4.4. Let $P$ be a finite p-group and let $\mathcal{Y}$ be a class of finite p-groups closed under taking sections and isomorphisms. Suppose that for any subgroup $Q$ of $P$ the map Defres $\mathcal{Y}$ : $D(Q) \rightarrow D\left(Q_{\mathcal{Y}}\right)$ is injective. Then, for any fusion system $\mathcal{F}$ on $P$, the diagram

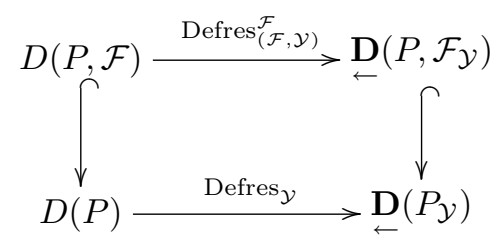

is a pull-back diagram, where the vertical maps are the canonical inclusions.

Proof. The commutativity of the diagram is immediate. By the assumptions, the bottom map Defres $_{\mathcal{Y}}$ in the diagram is injective. Since the vertical maps are inclusions, the top horizontal map $\operatorname{Defres}_{(\mathcal{F}, \mathcal{Y})}^{\mathcal{F}}$ is injective. We identify $D(P, \mathcal{F})$ and $\underset{\leftarrow}{\mathbf{D}}\left(P, \mathcal{F}_{\mathcal{Y}}\right)$ with their canonical images in $D(P)$ and $\mathbf{D}\left(P_{\mathcal{Y}}\right)$, respectively. Let $v \in D(P)$ such that $\operatorname{Defres}_{\mathcal{Y}}(v) \in \mathbf{D}\left(P, \mathcal{F}_{\mathcal{Y}}\right)$. We need to show that $v \in D(P, \mathcal{F})$. Write $\operatorname{Defres}_{\mathcal{Y}}(v)=\left(v_{(T, S)}\right)_{(T, S) \in P_{\mathcal{Y}}}$, with $v_{(T, S)}=\operatorname{Defres}_{T / S}^{P}(v)$, for all $(T, S) \in P_{\mathcal{Y}}$. Since $\operatorname{Defres}_{\mathcal{Y}}(v)$ is assumed to be an element of $\mathbf{D}\left(P, \mathcal{F}_{\mathcal{Y}}\right)$, we have $v_{(T, S)}=\operatorname{Res}_{\varphi}\left(v_{\left(T^{\prime}, S^{\prime}\right)}\right)$ for any morphism $\varphi:(T, S) \rightarrow\left(T^{\prime}, S^{\prime}\right)$ in $\mathcal{F}_{\mathcal{Y}}$. Let $Q$ be a subgroup of $P$ and let $\alpha \in \operatorname{Aut}_{\mathcal{F}}(Q)$, viewed as morphism from $Q$ to $P$. In order to show that $v \in D(P, \mathcal{F})$ it suffices to show that $\operatorname{Res}_{Q}^{P}(v)=\operatorname{Res}_{\text {alpha }}(v)$. The automorphism $\alpha$ of $Q$ induces an automorphism of the category $Q \mathcal{Y}$ and hence an automorphism of the group $\underset{\leftarrow}{\mathbf{D}}\left(Q_{\mathcal{Y}}\right)$, mapping an element $\left(w_{(T, S)}\right)_{(T, S) \in Q \mathcal{Y}}$ to $\left(\operatorname{Res}_{\alpha}\left(w_{(\alpha(T), \alpha(S))}\right)\right)_{(T, S) \in Q_{\mathcal{Y}}}$. This automorphism fixes $\left(v_{(T, S)}\right)_{(T, S) \in Q \mathcal{Y}}$ by the choice of $v$. Since the map Defresy $: D(Q) \rightarrow \mathbf{D}\left(Q_{\mathcal{Y}}\right)$ is injective it follows that $\alpha$ fixes $\operatorname{Res}_{Q}^{P}(v)$, or equivalently, $\operatorname{Res}_{Q}^{P}(v)=\operatorname{Res}_{\alpha}(v)$, whence the result.

Bouc and Thévenaz proved in [9, Theorem 4.7] that if $p$ is an odd prime and $\mathcal{X}$ is the class of all $p$-groups of order at most $p^{3}$ and exponent $p$, then for any finite $p$-group $P$ the group homomorphism Defres $_{\mathcal{X}}: D(P) \rightarrow \underset{\leftarrow}{\mathbf{D}}\left(P_{\mathcal{X}}\right)$ is an isomorphism. Combining this with Proposition 4.4 yields the following consequence.

Corollary 4.5. Let $p$ be an odd prime and let $\mathcal{X}$ be the class of all p-groups of order at most $p^{3}$ and exponent at most $p$. For any finite p-group $P$ and any fusion system $\mathcal{F}$ on $P$ the map

$$
\operatorname{Defres}_{(\mathcal{F}, \mathcal{X})}^{\mathcal{F}}: D(P, \mathcal{F}) \rightarrow \underset{\leftarrow}{\mathbf{D}}\left(P, \mathcal{F}_{\mathcal{X}}\right)
$$


is an isomorphism.

Remark 4.6. Using 4.5 it is easy to check that the group $\underset{\leftarrow}{\mathbf{D}}\left(P, P_{\mathcal{X}}^{\mathbf{1}}\right)$ is isomorphic to the subgroup of $\bigoplus_{1<Q \leq P} D\left(N_{P}(Q) / Q\right)$ defined by Bouc and Thévenaz at the beginning of $[8, \S 2]$.

\section{Gluing}

"Gluing problems" ask which families $\left(v_{(T, S)}\right)_{(T, S) \in \mathcal{C}_{\mathcal{Y}}}$ are in the image of the map

$$
\operatorname{Defres}_{(\mathcal{C}, \mathcal{Y})}^{\mathcal{F}}: D(P) \longrightarrow \prod_{(T, S) \in \mathcal{C}_{\mathcal{Y}}} D(T / S) .
$$

where the notation is as in the previous section. A subcategory $\mathcal{C}$ of a category $\mathcal{F}$ is called convex if $\mathcal{C}$ is a full subcategory of $\mathcal{F}$ with the property that whenever $X, Y, Z$ are three objects in $\mathcal{F}$ such that the morphism sets $\operatorname{Hom}_{\mathcal{F}}(X, Y), \operatorname{Hom}_{\mathcal{F}}(Y, Z)$ are non-empty and such that $X, Z$ belong to the subcategory $\mathcal{C}$, then also $Y$ belongs to $\mathcal{C}$. A convex subcategory is closed under isomorphisms.

Proposition 5.1. Let $p$ be an odd prime, let $P$ be a finite $p$-group, let $\mathcal{F}$ be a fusion system on $P$ and let $\mathcal{C}$ be a convex subcategory of $\mathcal{F}$. Let $\mathcal{Y}$ be a class of finite p-groups closed under taking sections and isomorphisms and containing the class $\mathcal{X}$ of $p$-subgroups of order at most $p^{3}$ and exponent at most $p$. The inclusion $\mathcal{C}_{\mathcal{X}} \subseteq \mathcal{C}_{\mathcal{Y}}$ induces an isomorphism

$$
\underset{\leftarrow}{\mathbf{D}}\left(P, \mathcal{C}_{\mathcal{Y}}\right) \cong \underset{\leftarrow}{\mathbf{D}}\left(P, \mathcal{C}_{\mathcal{X}}\right)
$$

Proof. Identify $\underset{\leftarrow}{\mathbf{D}}\left(P, \mathcal{C}_{\mathcal{Y}}\right)$ and $\underset{\leftarrow}{\mathbf{D}}\left(P, \mathcal{C}_{\mathcal{X}}\right)$ with the families $\left(v_{(T, S)}\right)_{(T, S)}$ satisfying

$$
\operatorname{Defres}_{\varphi}\left(v_{\left(T^{\prime}, S^{\prime}\right)}\right)=v_{(T, S)}
$$

for all objects $(T, S)$ and morphisms $\varphi$ belonging to the categories $\mathcal{C}_{\mathcal{Y}}$ and $\mathcal{C}_{\mathcal{X}}$, respectively. The inclusion $\mathcal{C}_{\mathcal{X}} \subseteq \mathcal{C}_{\mathcal{Y}}$ induces a canonical morphism

$$
\underset{\leftarrow}{\mathbf{D}}\left(P, \mathcal{C}_{\mathcal{Y}}\right) \rightarrow \underset{\leftarrow}{\mathbf{D}}\left(P, \mathcal{C}_{\mathcal{X}}\right)
$$

We use Corollary 4.5 to show that this is an isomorphism. We construct an inverse as follows. Let $\left(v_{(T, S)}\right)_{(T, S) \in \mathcal{C}_{\mathcal{X}}}$ be an element in $\underset{\leftarrow}{\mathbf{D}}\left(P, \mathcal{C}_{\mathcal{X}}\right)$. Let $(R, Q)$ be an object in $\mathcal{C}_{\mathcal{Y}}$. Note that since $\mathcal{C}$ is convex, the objects of $(R / Q)_{\mathcal{X}}$ belong to the category $\mathcal{C}_{\mathcal{X}}$. Indeed, let $(T / Q, S / Q) \in(R / Q)_{\mathcal{X}}$. That is, we have a chain $Q \unlhd S \unlhd T \leq R$ of objects of $\mathcal{F}$, with $Q \unlhd R$ and $R / Q \in \mathcal{Y}$. Thus $S, T \in \mathcal{C}$ since $Q, R \in \mathcal{C}$ and $\mathcal{C}$ is convex. Consequently, $(T / Q, S / Q) \cong(T, S) \in \mathcal{C}_{\mathcal{X}}$. Now, define $w_{(R, Q)} \in D(R / Q)$ as pre-image of the subfamily $\left(v_{(T, S)}\right)_{(T, S) \in(R / Q)_{\mathcal{X}}}$ under the isomorphism

$$
\text { Defres }_{\mathcal{X}}: D(R / Q) \longrightarrow \underset{\leftarrow}{\mathbf{D}}((R / Q) \mathcal{X})
$$

from [9, Theorem 4.7]. The family $\left(w_{(R, Q)}\right)_{(R, Q) \in \mathcal{C}_{\mathcal{Y}}}$ defined in this way belongs to $\underset{\leftarrow}{\mathbf{D}}\left(P, \mathcal{C}_{\mathcal{Y}}\right)$. In particular, $w_{(T, S)}=v_{(T, S)}$, for all $(T, S) \in \mathcal{C}_{\mathcal{X}}$, whence the composition

$$
\underset{\leftarrow}{\mathbf{D}}\left(P, \mathcal{C}_{\mathcal{X}}\right) \rightarrow \underset{\leftarrow}{\mathbf{D}}\left(P, \mathcal{C}_{\mathcal{Y}}\right) \rightarrow \underset{\leftarrow}{\mathbf{D}}\left(P, \mathcal{C}_{\mathcal{X}}\right)
$$

is the identity. For the composition of the maps the other way round, let $\left(v_{(R, Q)}\right)_{(R, Q) \in \mathcal{C}_{\mathcal{Y}}} \in$ $\mathbf{D}\left(P, \mathcal{C}_{\mathcal{Y}}\right)$, and let $\left(w_{(R, Q)}\right)_{(R, Q) \in \mathcal{C}_{\mathcal{Y}}}$ be the image of $\left(v_{(R, Q)}\right)_{(R, Q) \in \mathcal{C}_{\mathcal{X}}} \in \mathbf{D}\left(P, \mathcal{C}_{\mathcal{X}}\right)$ under the map 
defined above. Identify, via $\operatorname{Defres}_{\mathcal{X}}$, any $w_{(R, Q)} \in D(R / Q)$ with

$$
\left(\operatorname{Defres}_{T / S}^{R / Q} w_{(R, Q)}\right)_{(T, S) \in(R / Q)_{\mathcal{X}}} \in \underset{\leftarrow}{\mathbf{D}}\left((R / Q)_{\mathcal{X}}\right), \forall(R, Q) \in \mathcal{C}_{\mathcal{Y}} .
$$

By construction of $w_{(R, Q)} \in D(R / Q)$, we have, for any $(T, S) \in \mathcal{C}_{\mathcal{X}}$,

$$
\operatorname{Defres}_{T / S}^{R / Q} w_{(R, Q)}=w_{(T, S)}=v_{(T, S)} .
$$

Thus, Defres ${ }_{\mathcal{X}}$ associates any $w_{(R, Q)} \in D(R / Q)$ with $\left(v_{(T, S)}\right)_{(T, S) \in(R / Q)_{\mathcal{X}}} \in \mathbf{D}\left((R / Q)_{\mathcal{X}}\right)$, which is identified with $v_{(R, Q)}$ under Defres ${ }_{\mathcal{X}}^{-1}$. Hence $v_{(R, Q)}=w_{(R, Q)}$, for all $(R, Q) \in \mathcal{C}_{\mathcal{Y}}$. It follows that the composition of maps

$$
\underset{\leftarrow}{\mathbf{D}}\left(P, \mathcal{C}_{\mathcal{Y}}\right) \rightarrow \underset{\leftarrow}{\mathbf{D}}\left(P, \mathcal{C}_{\mathcal{X}}\right) \rightarrow \underset{\leftarrow}{\mathbf{D}}\left(P, \mathcal{C}_{\mathcal{Y}}\right)
$$

yields the identity, as was left to be shown.

Definition 5.2. Let $p$ be a prime and let $P$ be a finite $p$-group. Denote by $\mathcal{A}_{\geq 2}(P)$ the partially ordered set of elementary abelian subgroups of $P$ of rank at least 2. For any fusion system $\mathcal{F}$ on $P$ denote by $\mathcal{E}_{\geq 2}(\mathcal{F})$ the full subcategory of $\mathcal{F}$ having $\mathcal{A}_{\geq 2}(P)$ as set of objects; if $\mathcal{F}=\mathcal{F}_{P}(P)$ we write $\mathcal{E}_{\geq 2}(P)$ instead of $\mathcal{E}_{\geq 2}\left(\mathcal{F}_{P}(P)\right)$. Denote by $\mathbb{E}_{\mathcal{F}}$ the quotient of the $\mathbb{F}_{2}$-vector space of maps $\mathcal{E}_{\geq 2}(\mathcal{F}) \rightarrow \mathbb{F}_{2}$ which are constant on connected components of $\mathcal{E}_{\geq 2}(\mathcal{F})$ by the subspace of constant maps $\mathcal{E}_{\geq 2}(\mathcal{F}) \rightarrow \mathbb{F}_{2} ;$ in the case $\mathcal{F}=\mathcal{F}_{P}(P)$ we write $\mathbb{E}_{P}$ instead of $\mathbb{E}_{\mathcal{F}_{P}(P)}$.

Bouc and Thévenaz proved in $[8$, Theorem 5.1] that for $p$ an odd prime and $P$ a non cyclic finite $p$-group there is a short exact sequence of $\mathbb{F}_{2}$-vector spaces

$$
0 \longrightarrow D^{t}(P) \stackrel{\operatorname{Defres}_{\left(P^{1}, \mathcal{X}\right)}^{P}}{\longrightarrow} \underset{\leftarrow}{\mathbf{D}^{t}}\left(P, P_{\mathcal{X}}^{\mathbf{1}}\right) \stackrel{\Phi_{P}}{\longrightarrow} \mathbb{E}_{P} \longrightarrow 0
$$

where $P^{1}$ is the full subcategory of $\mathcal{F}_{P}(P)$ consisting of all non-trivial subgroups of $P$. In particular, if $\mathcal{A}_{\geq 2}(P)$ is connected we have an isomorphism $D^{t}(P) \cong \mathbf{D}^{t}\left(P, P_{\mathcal{X}}^{\mathbf{1}}\right)$. If $\mathcal{A}_{\geq 2}(P)$ is not connected, the map $\Phi_{P}$ is constructed explicitly, making use of the very particular structure of $P$ and the connected components of $\mathcal{A}_{\geq 2}(P)$ in that case. This can be generalised to arbitrary fusion systems; the proof is not as straightforward as one might expect:

Theorem 5.3. Let $p$ be an odd prime, $P$ a finite $p$-group and $\mathcal{F}$ a fusion system on $P$. Denote by $\mathcal{F}^{\mathbf{1}}$ the full subcategory of $\mathcal{F}$ consisting of all non-trivial subgroups of $P$ and let $\mathcal{X}$ be the class of finite p-groups of order at most $p^{3}$ and exponent at most $p$. The map $\Phi_{P}$ induces a short exact sequence of $\mathbb{F}_{2}$-vector spaces

$$
0 \longrightarrow D^{t}(P, \mathcal{F}) \longrightarrow \mathbb{D}^{t}\left(P, \mathcal{F}_{\mathcal{X}}^{\mathbf{1}}\right) \longrightarrow \mathbb{E}_{\mathcal{F}} \longrightarrow 0
$$

As in the proof of [8, Theorem 5.1], the difficult part of the proof of 5.3 arises when $\mathcal{A}_{\geq 2}(P)$ is not connected. By [12, Lemma 2.2], in that case, $P$ has a unique central cyclic subgroup of order $p$, which we will denote by $Z$. Furthermore, any connected component of $\mathcal{A}_{\geq 2}(P)$ contains either all elementary abelian subgroups of rank at least 3 or consists of a single elementary abelian subgroup $E$ of rank 2 , in which case $E$ is called isolated in $\mathcal{A}_{\geq 2}(P)$. If $P$ has rank at least 3 we denote by $\mathcal{B}$ the connected component of $\mathcal{A}_{\geq 2}(P)$ containing all elementary abelian subgroups of 
rank at least 3 (and their subgroups of rank 2), and in that case, $\mathcal{B}$ contains a subgroup $E_{0}$ of rank 2 which is normal in $P$. If $P$ has rank 2 , any connected component of $\mathcal{A}_{\geq 2}(P)$ consists of a single isolated subgroup of rank 2 , and there exists an isolated $E_{0}$ which is normal in $P$; we set $\mathcal{B}=\left\{E_{0}\right\}$ in that case. Whenever $E$ is isolated in $\mathcal{A}_{\geq 2}(P)$ then $E$ contains $Z$, the group $N_{P}(E)$ acts transitively on the set of complements of $Z$ in $E$, and for any such complement $S$ of $Z$ in $E$ we have $N_{P}(S)=S \times L$ for some cyclic subgroup $L$ of $P$. One of the additional difficulties in the proof of 5.3 is that $Z$ need no longer be strongly $\mathcal{F}$-closed - and hence a connected component of $\mathcal{E}_{\geq 2}(\mathcal{F})$ may contain both isolated and non isolated elementary abelian subgroups of $P$. The following lemma will be needed to handle this situation.

Lemma 5.4. Let $p$ be an odd prime, $P$ a finite $p$-group and $\mathcal{F}$ a fusion system on $P$. Let $E$ be an elementary abelian subgroup of rank 2 of $P$ which is isolated in $\mathcal{A}_{>2}(P)$ and let $Q$ be a subgroup of order $p$ of $E$. Let $\psi \in \operatorname{Hom}_{\mathcal{F}}(E, P)$. If there is an automorphism $\alpha \in \operatorname{Aut}_{\mathcal{F}}(E)$ such that $\alpha(Q) \neq Z$ then there is an automorphism $\beta \in \operatorname{Aut}_{\mathcal{F}}(\psi(E))$ such that $\beta(\psi(Q)) \neq Z$.

Proof. Note that $E$ contains $Z$ by the above remarks. If $\psi(Q) \neq Z$ we take $\beta=\operatorname{Id}_{\psi(E)}$. Suppose that $\psi(Q)=Z$. If also $Q=Z$ take $\beta=\psi \circ \alpha \circ \psi^{-1}$. If $Q \neq Z$ there is $y \in N_{P}(E)$ such that ${ }^{y} Q \neq Q$ because $N_{P}(E)$ acts transitively on the set of complements of $Z$ in $E$, again by the above remarks. Thus in this case take $\beta=\psi \circ c_{y} \circ \psi^{-1}$, where $c_{y}$ is the automorphism of $E$ given by conjugation with $y$.

Proof of Theorem 5.3. We identify $D^{t}(P, \mathcal{F})$ and $\mathbf{D}^{t}\left(P, \mathcal{F}_{\mathcal{X}}^{\mathbf{1}}\right)$ with their canonical images in $D^{t}(P)$ and $\mathbf{D}^{t}\left(P, P_{\mathcal{X}}^{\mathbf{1}}\right)$, respectively. Clearly the map $\overleftarrow{D^{t}}(P) \rightarrow \mathbf{D}^{t}\left(P, P_{\mathcal{X}}^{\mathbf{1}}\right)$ induces an injective map $D^{t}(P, \mathcal{F}) \rightarrow \mathbf{D}^{t}\left(P, \mathcal{F}_{\mathcal{X}}^{\mathbf{1}}\right)$. We break up the proof in several steps.

We show the exactness at $\mathbf{D}^{t}\left(P, \mathcal{F}_{\mathcal{X}}^{\mathbf{1}}\right)$. Let $\left(v_{(T, S)}\right)_{(T, S) \in \mathcal{F}_{\mathcal{X}}^{1}}$ be an element in $\operatorname{ker}\left(\Phi_{P}\right) \cap \mathbf{D}^{t}\left(P, \mathcal{F}_{\mathcal{X}}^{\mathbf{1}}\right)$. Explicitly, this means that for any morphism $\varphi:(T, S) \rightarrow\left(T^{\prime}, S^{\prime}\right)$ in $\mathcal{F}_{\mathcal{X}}^{\mathbf{1}}$ we have an equality $v_{(T, S)}=\operatorname{Defres}_{\varphi}\left(v_{\left(T^{\prime}, S^{\prime}\right)}\right)$, where $\operatorname{Defres}_{\varphi}$ is as defined in 4.2. The fact that this element is also in $\operatorname{ker}\left(\Phi_{P}\right)$ implies, by the above exact sequence of Bouc and Thévenaz, that there is an element $v \in D^{t}(P)$ such that $\operatorname{Defres}_{T / S}^{P}(v)=v_{(T, S)}$ for any object $(T, S)$ in $\mathcal{F}_{\mathcal{X}}^{\mathbf{1}}$. We need to show that $v$ belongs actually to $D^{t}(P, \mathcal{F})$. By Alperin's fusion theorem, we need to show that for any $\mathcal{F}$ centric subgroup $Q$ of $P$ and any $\varphi \in \operatorname{Aut}_{\mathcal{F}}(Q)$ considered as morphism from $Q$ to $P$ we have $\operatorname{Res}_{Q}^{P}(v)=\operatorname{Res}_{\varphi}(v)$ in $D^{t}(Q)$. If $Q$ is cyclic this is trivial. If $Q$ is not cyclic, the exact sequence of Bouc and Thévenaz applies and yields in particular an injective map $D^{t}(Q) \rightarrow \mathbf{D}^{t}\left(Q, Q_{\mathcal{X}}^{\mathbf{1}}\right)$. In other words, $\operatorname{Res}_{\varphi}(v)$ is completely determined by the family $\left(\operatorname{Defres}_{V / U}^{Q}\left(\operatorname{Res}_{\varphi}(v)\right)\right)_{(V, U) \in Q_{\mathcal{X}}^{1}}$. Now, by transitivity of restriction, $\operatorname{Defres}_{V / U}^{Q}\left(\operatorname{Res}_{Q}^{P} v\right)=\operatorname{Defres}_{V / U}^{P}(v)=v_{(V, U)}$. Moreover, by assumption on $v$, we have $v_{(V, U)}=\operatorname{Res}_{\varphi}\left(v_{(\varphi(V), \varphi(U))}\right)$. Thus, the equality $\operatorname{Res}_{Q}^{P}(v)=\operatorname{Res}_{\varphi}(v)$ holds. This shows the exactness at $\mathbf{D}^{t}\left(P, \mathcal{F}_{\mathcal{X}}^{\mathbf{1}}\right)$.

We show next the exactness at $\mathbb{E}_{\mathcal{F}}$. If $\mathcal{A}_{\geq 2}(P)$ is connected then $\mathbb{E}_{P}=\{0\}$, hence $\mathbb{E}_{\mathcal{F}}=$ $\{0\}$, and we get an isomorphism $D^{t}(P, \mathcal{F}) \cong \overline{\mathbf{D}}^{t}\left(P, \mathcal{F}_{\mathcal{X}}^{\mathbf{1}}\right)$, which proves the theorem in that case. We assume now that $\mathcal{A}_{\geq 2}(P)$ is not connected, and so $P$ has the particular structure described above. The proof of the exactness at $\mathbb{E}_{\mathcal{F}}$ breaks up into two parts: we first need to show that $\Phi_{P}$ 
maps $\mathbb{D}^{t}\left(P, \mathcal{F}_{\mathcal{X}}^{\mathbf{1}}\right)$ to $\mathbb{E}_{\mathcal{F}}$, and second that this is onto. Let $v=\left(v_{(T, S)}\right)_{(T, S) \in \mathcal{F}_{\mathcal{X}}^{1}}$ be an element in $\mathbf{D}^{t}\left(P, \overleftarrow{\mathcal{F}}_{\mathcal{X}}^{\mathbf{1}}\right)$. The map $\Phi_{P}$ in [8, Theorem 5.1] is constructed as follows: for $E$ an elementary abelian subgroup of $P$ of rank at least 2 , the subfamily $\left(v_{(T, S)}\right)_{(T, S) \in(E Z)_{X}^{1}}$ determines a unique element $w_{E Z}$ in $D^{t}(E Z)$, where we use the isomorphism $D^{t}(E Z) \cong \mathbf{D}^{t}\left(E Z,(E Z)_{\mathcal{X}}^{1}\right)$ from [8, Theorem 5.1] applied to the elementary abelian group $E Z$. Since $Z$ is cyclic of order $p$ we have $D(Z) \cong \mathbb{F}_{2}$. Thus, restriction from $E Z$ to $Z$ sends $w_{E Z}$ to a uniquely determined element $\epsilon_{v}(E) \in \mathbb{F}_{2}$. It is shown in [8, Lemma 3.2] that this defines a map $\epsilon_{v}$ on $\mathcal{A}_{\geq 2}(P)$ satisfying $\epsilon_{v}(E)=\epsilon_{v}\left(E^{\prime}\right)$ if $E$ is conjugate to a subgroup of $E^{\prime}$, and hence $\epsilon_{v}$ is in fact a map $\mathcal{E}_{\geq 2}(P) \rightarrow \mathbb{F}_{2}$ which is constant on connected components of $\mathcal{E}_{\geq 2}(P)$. That is, $\epsilon_{v}$ yields an element $\bar{\epsilon}_{v}$ in $\mathbb{E}_{P}$. We need to show that $\bar{\epsilon}_{v}$ belongs to $\mathbb{E}_{\mathcal{F}}$. It suffices to show that the map $\epsilon_{v}: \mathcal{E}_{\geq 2}(P) \rightarrow \mathbb{F}_{2}$ is constant on connected components of $\mathcal{E}_{\geq 2}(\mathcal{F})$. Since $\epsilon_{v}$ is invariant with respect to inclusions, it suffices to show that $\epsilon_{v}(E)=\epsilon_{v}(\varphi(E))$, for all $\varphi \in \operatorname{Hom}_{\mathcal{F}}(E, P)$, with $E$ elementary abelian of rank 2. If $E$ and $\varphi(E)$ are in the same connected component of $\mathcal{E}_{\geq 2}(P)$ the equality holds by [8, Theorem 5.1]. Thus we may assume that $E$ is isolated. Write $\bar{E}=S \times Z$, where $Z$ is the unique central subgroup of order $p$. Then $N_{P}(S)=C_{P}(E)=S \times L$ has rank 2 as well. Consider first the case where $\varphi(E)$ is also isolated for all $\varphi \in \operatorname{Hom}_{\mathcal{F}}(E, P)$. Choose $\varphi \in \operatorname{Hom}_{\mathcal{F}}(E, P)$ such that $\varphi(E)$ is fully $\mathcal{F}$-normalised, so that $N_{\varphi}=N_{P}(E)$; that is, such that $\varphi$ extends to a morphism $\tilde{\varphi}: N_{P}(E) \rightarrow P$. Since $N_{P}(E)$ permutes transitively the complements of $Z$ in $E$, the group $\tilde{\varphi}\left(N_{P}(E)\right.$ ), which is contained in $N_{P}(\varphi(E))$, permutes transitively the complements of $\varphi(Z)$ in $\varphi(E)$. On the other hand, $\varphi(E)=Z \times S^{\prime}$ for some non-central subgroup $S^{\prime}$ of $P$ of order $p$, and $N_{P}(\varphi(E))$ permutes transitively the complements of $Z$ in $\varphi(E)$. This forces $\varphi(Z)=Z$. It follows, by definition of $\Phi_{P}$, that $w_{E}=\operatorname{Res}_{\varphi}\left(w_{\varphi(E)}\right)$, and hence $\epsilon_{v}(E)=\epsilon_{v}(\varphi(E))$. Since there is always an isomorphism $\varphi$ with $N_{\varphi}=N_{P}(E)$, we conclude that $\epsilon_{v}(E)=\epsilon_{v}(\varphi(E))$, for all $\varphi \in \operatorname{Hom}_{\mathcal{F}}(E, P)$, in the situation where $\varphi(E)$ is isolated for all $\varphi \in \operatorname{Hom}_{\mathcal{F}}(E, P)$. Suppose next that there exists $\varphi \in \operatorname{Hom}_{\mathcal{F}}(E, P)$ such that $\varphi(E)$ is contained in an elementary abelian subgroup of rank 3. Denote by $\psi: \varphi(Z) \rightarrow Z$ the restriction of the inverse of $\varphi$. Clearly, $Z$ is fully $\mathcal{F}$-centralised, and hence $\psi$ extends to a morphism $\tilde{\psi}: C_{P}(\varphi(Z)) \rightarrow P$, such that the composition $\tilde{\psi} \circ \varphi$ is the identity on $Z$. Note that $\varphi(E) \leq C_{P}(\varphi(E)) \leq C_{P}(\varphi(Z))$ and that $\tilde{\psi}$ maps $C_{P}(\varphi(E))$ to $C_{P}(\tilde{\psi}(\varphi(E)))$, which has rank at least 3. Thus, $\tilde{\psi}(\varphi(E))$ belongs to the connected component $\mathcal{B}$ of $\mathcal{A}_{\geq 2}(P)$. As before, the equality $\tilde{\psi}(\varphi(Z))=Z$ implies $w_{E}=\operatorname{Res}_{\varphi}\left(w_{\varphi(E)}\right)$, hence $\epsilon_{v}(E)=\epsilon_{v}(\tilde{\psi}(\varphi(E))$. In other words, the value of $\epsilon_{v}$ at $E$ coincides with the value of $\epsilon_{v}$ on $\mathcal{B}$. This shows that $\Phi_{P}$ maps $\mathbf{D}^{t}\left(P, \mathcal{F}_{\mathcal{X}}^{1}\right)$ to $\mathbb{E}_{\mathcal{F}}$, by $\Phi_{P}(v)=\epsilon_{v}$.

In order to show that $\Phi_{P}$ maps $\underline{\mathbf{D}}^{t}\left(P, \mathcal{F}_{\mathcal{X}}^{1}\right)$ onto $\mathbb{E}_{\mathcal{F}}$, we define a map $\sigma: \mathbb{E}_{\mathcal{F}} \rightarrow \underline{\mathbf{D}}^{t}\left(P, \mathcal{F}_{\mathcal{X}}^{1}\right)$, such that $\Phi_{P} \circ \sigma$ is the identity on $\mathbb{E}_{\mathcal{F}}$. Let $c$ be a connected component of $\mathcal{E}_{>2}(\mathcal{F})$. Write $\epsilon_{c}: \mathcal{E}_{\geq 2}(\mathcal{F}) \rightarrow \mathbb{F}_{2}$ for the map taking values $\epsilon_{c}(E)=1$ if $E \in c$ and $\epsilon_{c}(E)=0$ otherwise. The image $\bar{B}=\left\{\bar{\epsilon}_{c} \mid \mathcal{B} \nsubseteq \subset c\right\}$ under the canonical projection of the set $B=\left\{\epsilon_{c} \mid \mathcal{B} \nsubseteq c\right\}$, where $c$ runs through the connected components of $\mathcal{E}_{>2}(\mathcal{F})$ not containing $\mathcal{B}$, forms an $\mathbb{F}_{2}$-basis of $\mathbb{E}_{\mathcal{F}}$. If $\mathcal{E}_{\geq 2}(\mathcal{F})$ is connected, or equivalently, if $\mathbb{E}_{\mathcal{F}}=\{0\}$ there is nothing to prove. Assume that $\mathbb{E}_{\mathcal{F}} \neq\{0\}$ and let $c$ be a connected component in $\mathcal{E}_{\geq 2}(\mathcal{F})$ not containing $\mathcal{B}$; that is, $c$ consists of subgroups of rank 2 which are isolated in $\mathcal{A}_{\geq 2}(P)$. For any object $(R, Q)$ in $\mathcal{F}_{\mathcal{X}}^{\mathbf{1}}$ set

$$
\mathcal{N}(c, R, Q)=\left\{E \in c \mid Q<E \leq R ; \exists \varphi \in \operatorname{Aut}_{\mathcal{F}}(E): \varphi(Q) \neq Z\right\}
$$


and define an element in $D^{t}(R / Q)$ by setting

$$
\sigma\left(\bar{\epsilon}_{c}\right)_{(R, Q)}=\sum_{E \in \mathcal{N}(c, R, Q)} \operatorname{Ten}_{E / Q}^{R / Q}\left(\Omega_{E / Q}\right)
$$

with the convention $\sigma\left(\bar{\epsilon}_{c}\right)_{(R, Q)}=0$ if the set $\mathcal{N}(c, R, Q)$ is empty. Note that if $\mathcal{N}(c, R, Q)$ is non empty then $Q$ has order $p$. We will show that the family

$$
\sigma\left(\bar{\epsilon}_{c}\right)=\left(\sigma\left(\bar{\epsilon}_{c}\right)_{(R, Q)}\right)_{(R, Q) \in \mathcal{F}_{\mathcal{X}}^{1}}
$$

is an element in the group $\mathbf{D}^{t}\left(P, \mathcal{F}_{\mathcal{X}}^{\mathbf{1}}\right)$; that is, given a morphism $\psi:(R, Q) \rightarrow\left(R^{\prime}, Q^{\prime}\right)$ in $\mathcal{F}_{\mathcal{X}}^{\mathbf{1}}$ we need to show that

$$
\operatorname{Defres}_{\psi}\left(\sigma\left(\bar{\epsilon}_{c}\right)_{\left(R^{\prime}, Q^{\prime}\right)}\right)=\sigma\left(\bar{\epsilon}_{c}\right)_{(R, Q)}
$$

If $Q, Q^{\prime}$ have order greater than $p$ both sides are zero, so we may assume that $Q^{\prime}$ has order $p$. If $Q$ has order greater than $p$ then again both sides are zero. Thus we may assume that $\psi(Q)=Q^{\prime}$. We consider first the case where $\psi(R)=R^{\prime}$; that is, $\psi$ is an isomorphism in $\mathcal{F}_{\mathcal{X}}^{\mathbf{1}}$. In that case, thanks to $5.4, \psi$ induces a bijection between the sets $\mathcal{N}(c, R, Q)$ and $\mathcal{N}\left(c, R^{\prime}, Q^{\prime}\right)$, and thus the required equality follows. We may therefore assume, after possibly replacing $(R, Q)$ by $(\psi(R), \psi(Q))$, that $Q=Q^{\prime} \leq R<R^{\prime}$ and that $\psi$ is the obvious inlcusion map. We show that this implies $Q=Z$. Indeed, if $Q \neq Z$ there is at most one isolated $E$ containing $Q$, namely $E=Q \times Z$. Thus either $\mathcal{N}(c, R, Q), \mathcal{N}\left(c, R^{\prime}, Q\right)$ are both empty (in which case we are done) or both equal to $\{E\}$, where $E=Q \times Z$. But in that case we have $R^{\prime} \leq N_{P}(Q)=Q \times L$ for some cyclic $L \leq P$. Since also $R^{\prime} / Q$ has exponent at most $p$, this forces $E=R=R^{\prime}$, contradicting our current assumption $R<R^{\prime}$. Thus $Q=Q^{\prime} Z$, and Defres $\psi_{\psi}$ is just restriction $\operatorname{Res}_{R / Z}^{R^{\prime} / Z}$ from $R^{\prime} / Z$ to $R / Z$. Thus

$$
\operatorname{Defres}_{\psi}\left(\sigma\left(\bar{\epsilon}_{c}\right)_{\left(R^{\prime}, Z\right)}\right)=\sum_{F \in \mathcal{N}\left(c, R^{\prime}, Z\right)} \operatorname{Res}_{R / Z}^{R^{\prime} / Z} \operatorname{Ten}_{F / Z}^{R^{\prime} / Z}\left(\Omega_{F / Z}\right)
$$

Note that if ${ }^{x} F$ is not contained in $R$ then $R / Z \cap{ }^{x} F / Z$ is trivial because $F$ has order $p^{2}$. Thus the Mackey formula applied to the summands in the above expression yields

$$
\operatorname{Defres}_{\psi}\left(\sigma\left(\bar{\epsilon}_{c}\right)_{\left(R^{\prime}, Z\right)}\right)=\sum_{(F, x) \in \mathcal{A}} \operatorname{Ten}_{x / Z}^{R / Z}\left(\Omega_{x} F / Z\right)
$$

where $\mathcal{A}$ is the set of all pairs $(F, x)$ in $\mathcal{N}\left(c, R^{\prime}, Z\right) \times\left[R \backslash R^{\prime}\right]$ satisfying ${ }^{x} F \leq R$. Now ${ }^{x} F \leq R$ means precisely that ${ }^{x} F \in \mathcal{N}(c, R, Z)$. Thus the summands on the right side of the previous expression are of the form $\operatorname{Ten}_{E / Z}^{R / Z}\left(\Omega_{E / Z}\right)$ for some $E \in \mathcal{N}(c, R, Z)$. In order to check that this sum is equal to $\sigma\left(\bar{\epsilon}_{c}\right)_{(R, Z)}$, all we need to do is to check that the multiplicity of such a summand is odd because they all have order two in the Dade group. Write $\mathcal{A}$ as disjoint union of sets $\mathcal{A}_{E}$, where, for $E \in \mathcal{N}(c, R, Q)$, we set

$$
\mathcal{A}_{E}=\left\{(F, x) \in \mathcal{A} \mid{ }^{x} F=E\right\}
$$

and denote by $a_{E}$ the cardinal of $\mathcal{A}_{E}$. Then

$$
\operatorname{Defres}_{\psi}\left(\sigma\left(\bar{\epsilon}_{c}\right)_{\left(R^{\prime}, Z\right)}\right)=\sum_{E \in \mathcal{N}(c, R, Q)} a_{E} \cdot \operatorname{Ten}_{E / Z}^{R / Z}\left(\Omega_{E / Z}\right)
$$

We need to show that the coefficients $a_{E}$ are odd, for $E \in \mathcal{N}(c, R, Z)$. Every element $(F, x)$ in $\mathcal{A}_{E}$ is uniquely determined by its second component $x \in\left[R \backslash R^{\prime}\right]$ because ${ }^{x} F=E$, hence $F={ }^{x^{-1}} E$. 
Thus $a_{E}=\left[R^{\prime}: R\right]$, which is a power of the odd prime $p$, hence odd. This concludes the proof of the fact that the family $\sigma\left(\bar{\epsilon}_{c}\right)$ belongs to the group $\mathbf{D}^{t}\left(P, \mathcal{F}_{\mathcal{X}}^{\mathbf{1}}\right)$.

Finally, we need to show that the map $\sigma: \mathbb{E}_{\mathcal{F}} \rightarrow \mathbf{D}^{t}\left(P, \mathcal{F}_{\mathcal{X}}^{1}\right)$ is a section for $\Phi_{P}$ restricted to $\mathbf{D}^{t}\left(P, \mathcal{F}_{\mathcal{X}}^{\mathbf{1}}\right)$. That is, with the notation above, we need to show that $\Phi_{P}\left(\sigma\left(\bar{\epsilon}_{c}\right)\right)$ is the image $\bar{\epsilon}_{c}$ in $\mathbb{E}_{\mathcal{F}}$ of the map $\epsilon_{c}$ sending $E \in c$ to 1 and any $E \in \mathcal{E}_{\geq 2}(\mathcal{F})-c$ to zero. Let $E$ be an elementary abelian subgroup of $P$ of rank at least 2 such that $E \notin c$ and let $(R, Q) \in(E Z)_{\mathcal{X}}^{1}$. Then the set $\mathcal{N}(c, R, Q)$ is empty; indeed, any $E^{\prime} \in \mathcal{N}(c, R, Q)$ would be contained in $R$, hence in $E Z$, implying that $E, E^{\prime}$ belong to the same connnected component in $\mathcal{E}_{\geq 2}(\mathcal{F})$. Thus $\sigma\left(\bar{\epsilon}_{c}\right)_{(R, Q)}=0$ for all $(R, Q) \in(E Z)_{\mathcal{X}}^{1}$, and hence this family corresponds to the zero element in $D^{t}(E Z)$ via the isomorphism $D^{t}(E Z) \cong \mathbf{D}^{t}\left(E Z,(E Z)_{\mathcal{X}}^{1}\right)$, hence its image in $D^{t}(Z)$ is zero. By the definition of $\Phi_{P}$ this means that $\Phi_{P}\left(\sigma\left(\bar{\epsilon}_{c}\right)\right)$ is the image of a map sending $E \in \mathcal{E}_{>2}(\mathcal{F})-c$ to zero. Let now $E \in c$. The non trivial objects of $E_{\mathcal{X}}^{1}$ are of the form $(E, Q)$, where $Q$ is a subgroup of order $p$ of $E$, and we have $\sigma\left(\bar{\epsilon}_{c}\right)_{(E, Q)}=\Omega_{E / Q}$. The image in $D^{t}(E)$ of this family under the isomorphism $D^{t}(E) \cong \mathbf{D}^{t}\left(E, E_{\mathcal{X}}^{1}\right)$ is equal to $\sum_{Q ; 1<Q<E} \Omega_{E / Q}$. The restriction to $Z$ of this sum is the element $p \Omega_{Z}=\Omega_{Z}$ in $D^{t}(Z)$ because $E$ has exactly $p$ subgroups of order $p$ different from $Z$ and because $p$ is odd. This concludes the proof of the fact that $\Phi_{P}\left(\sigma\left(\bar{\epsilon}_{c}\right)\right)=\bar{\epsilon}_{c}$, whence the result.

\section{EXAMPLES}

The Dade group of a arbitrary fusion system on a finite 2-group $P$ whose possible essential subgroups are Klein or quaternion (of order 8) group turns out to be equal to the Dade group $D(P)$ of $P$ itself. This includes the case of all cyclic, generalised quaternion, quasi- and semidihedral 2-groups (cf. [15, Satz I.14.9] for the definitions).

Theorem 6.1. Let $P$ be a finite 2 -group and $\mathcal{F}$ a fusion system on $P$. Assume that all $\mathcal{F}$-essential subgroups of $P$ are Klein four groups or quaternion groups of order 8 . Then $D(P, \mathcal{F})=D(P)$.

By Alperin's fusion theorem (cf. Remark 3.5) it suffices to show that for any $\mathcal{F}$-essential fully $\mathcal{F}$-normalised subgroup $Q$ of $P$ and any automorphism $\varphi \in \operatorname{Aut}_{\mathcal{F}}(Q)$ we have $\operatorname{Res}_{\varphi}(v)=\operatorname{Res}_{Q}^{P}(v)$ in $D(Q)$, for all $v \in D(P)$. The proof is based on this in conjunction with the following two observations:

Lemma 6.2. Let $P$ be a finite $p$-group, where $p$ is any prime, let $Q$ be a characteristic subgroup of $P$, and let $\varphi \in \operatorname{Aut}(P)$. Then, $\operatorname{Res}_{\varphi}\left(\Omega_{P / Q}\right)=\Omega_{P / Q}$.

Proof. By definition, $\operatorname{Res}_{\varphi}\left(\Omega_{P / Q}\right)=\Omega_{\varphi(P) / \varphi(Q)}=\Omega_{P / Q}$, since $Q$ is characteristic.

Lemma 6.3. If $Q$ is a Klein four group or a quaternion group of order 8 , then for any $w \in D(Q)$ and any $\varphi \in \operatorname{Aut}(Q)$ of odd order we have $\operatorname{Res}_{\varphi}(w)=w$.

Proof. By Lemma 6.2, the Lemma holds for $w=\Omega_{Q}$, and $w=\Omega_{Q / Z}$ in case $Q$ is quaternion of order 8 and $Z$ is its centre. It remains to consider the 'exotic' elements $v=[V] \in D(Q)$ for the Dade group of a quaternion group $Q$ and in case $k$ has cubic roots of unity. By [11, $\S 6]$, in that case $Q$ has exactly two isomorphism classes of indecomposable endo-trivial modules of dimension 5 , and hence an automorphism of odd order must stabilise both classes. 
Proof of Theorem 6.1. By the previous remarks, in order to show the $\mathcal{F}$-stability of all the elements of $D(P)$, it is enough to consider the $\mathcal{F}$-stability with respect to automorphisms $\varphi \in \operatorname{Aut}_{\mathcal{F}}(Q)$, for $Q$ a fully $\mathcal{F}$-normalised $\mathcal{F}$-essential subgroup of $P$. Stability with respect to conjugation by elements in $P$ holds trivially, and since $\operatorname{Aut}_{P}(Q)$ is a $\operatorname{Sylow}^{2}$-subgroup of $\operatorname{Aut}_{\mathcal{F}}(Q)$, we may suppose that $\varphi$ has odd order. Hence, if all $\mathcal{F}$-essential 2-subgroups of $P$ are Klein groups or quaternion groups of order 8, then the only automorphisms $\varphi$ of $Q$ of odd order have order 3. By Lemma $6.3, D(Q)$ is $\langle\varphi\rangle$-stable, and hence $\operatorname{Res}_{Q}^{P}(v)=\operatorname{Res}_{\varphi}(v)$ for all $v \in D(P)$, as was left to be shown.

Corollary 6.4. If $P$ is a 2-group that is either cyclic, dihedral, semi-dihedral, quasi-dihedral or generalised quaternion, and $\mathcal{F}$ is a fusion system on $P$, then $D(P, \mathcal{F})=D(P)$.

A similar statement for odd $p$ does not hold because $\Omega_{C_{p}} \neq 0$, whereas $\Omega_{C_{2}}=0$. More precisely:

Proposition 6.5. Let $p$ be an odd prime and $P$ be an elementary abelian p-group of order $p^{2}$. Let $\mathcal{F}$ be a fusion system on $P$ containing a non trivial automorphism $\varphi \in \operatorname{Aut}(P)$ of order dividing $p+1$. Then $D(P, \mathcal{F})$ is a proper subgroup of $D(P)$.

Proof. Let $C$ be a subgroup of $P$ of order $p$ such that $\varphi(C) \neq C$. Then, $\operatorname{Res}_{\varphi}\left(\Omega_{P / C}\right)=\Omega_{P / \varphi(C)} \neq$ $\Omega_{P / C}$.

Example 6.6. Let $p$ be an odd prime, let $G=\operatorname{PSL}(3, p)$, let $P$ be a Sylow- $p$-subgroup of $G$ and set $\mathcal{F}=\mathcal{F}_{P}(G)$. Then $P$ is an extraspecial $p$-group of order $p^{3}$ and exponent $p$, and its center $Z$ has order $p$. Set $E_{1}, \ldots, E_{p+1}$ for the $p+1$ elementary abelian subgroups of rank 2 , and write $E_{i}=S_{i} \times Z$, for some non central subgroup $S_{i}$ of $E_{i}$ of order $p$, and for all $1 \leq i \leq p+1$. By [6],

$$
D(P)=\left\langle\Omega_{P / Q} \mid 1 \leq Q<P\right\rangle=\operatorname{ker}\left(\operatorname{Def}_{P / Z}^{P}\right) \oplus \operatorname{Inf}_{P / Z}^{D}(P / Z) \cong \mathbb{Z}^{p+2} \oplus(\mathbb{Z} / 2)^{p+2} .
$$

More precisely, let

$$
e_{0}=\Omega_{P}, e_{i}=\Omega_{P / S_{i}}, 1 \leq i \leq p+1, e_{p+2}=\Omega_{P / Z} \text {, and } e_{i}=\Omega_{P / E_{i-p-2}}, p+3 \leq i \leq 2 p+3 .
$$

Then, $\operatorname{ker}\left(\operatorname{Def}_{P / Z}^{P}\right)$ is generated by $e_{i}, 0 \leq i \leq p+1$, subject to the relation $2 e_{0}=\sum_{i=1}^{p+1} 2 e_{i}$; whereas $\operatorname{Inf}_{P / Z}^{D}(P / Z)$ is generated by all the other $e_{i}$ 's, subject to the relations $2 e_{i}=0$, for all $p+3 \leq$ $i \leq 2 p+3$. Moreover, $T(P) \cong \mathbb{Z}^{p+1}$ is generated by $e_{0}$ and $2 e_{i}$, for $1 \leq i \leq p+1$. By Alperin's fusion theorem, the $\mathcal{F}$-stable elements are detected upon $\mathcal{F}$-stability under automorphisms of $P$, and of the $\mathcal{F}$-essential subgroups. Here, there are two elementary abelian subgroups of order $p^{2}$ that are $\mathcal{F}$-essential. We choose notation such that $E_{1}$ and $E_{2}$ are essential (then the poset of the non trivial $p$-subgroups of the quotient group $N_{G}\left(E_{i}\right) / E_{i} C_{G}\left(E_{i}\right)$ is not connected, for $\left.i=1,2\right)$. Moreover, $E_{3}, \ldots, E_{p+1}$ form a single $\mathcal{F}$-conjugacy class. The group $D(P, \mathcal{F})$ is generated by the elements

$$
f_{0}=e_{0}, f_{1}=e_{p+4}+e_{1}, f_{2}=e_{p+3}+e_{2}, f_{3}=\sum_{i=3}^{p+1} e_{i} \text { and } f_{4}=\sum_{i=p+5}^{2 p+3} e_{i},
$$

subject to the relations $2 f_{0}-\sum_{i=1}^{3} 2 f_{i}=0$ and $2 f_{4}=0$.

Example 6.7. Let $P$ be an extraspecial group of order $7^{3}$ and exponent 7. By [22], there is an exotic fusion system $\mathcal{F}$ on $P$, where the $\mathcal{F}$-centric $\mathcal{F}$-radical subgroups of $P$ are the 8 elementary abelian subgroups of order 49. In the same notation as above, with $p=7$, we may assume that $E_{1}$ and $E_{2}$ are $\mathcal{F}$-conjugate, and $E_{3}, \ldots, E_{8}$ form a single $\mathcal{F}$-conjugacy class. Moreover, any 
morphism in $\mathcal{F}$ preserves $Z$. By $[6], D(P) \cong \mathbb{Z}^{9} \oplus(\mathbb{Z} / 2)^{9}$, and $T(P) \cong \mathbb{Z}^{3}$. A direct computation yields $D(P, \mathcal{F}) \cong \mathbb{Z}^{3} \oplus(\mathbb{Z} / 2)^{3}$, and also $T(P, \mathcal{F})=T(P)$, because $Z$ is normalised by $\mathcal{F}$. Explicitly, $\operatorname{ker}\left(\operatorname{Def}_{P / Z}^{P}\right) \cap D(P, \mathcal{F}) \cong \mathbb{Z}^{2}$ is generated by $e_{0}, e_{1}+e_{2}$ and $e_{3}+\cdots+e_{8}$ subject to the relation $2 e_{0}=\sum_{i=1}^{8} 2 e_{i}$, and $\operatorname{Inf}_{P / Z}^{P}(P / Z) \cap D(P, \mathcal{F}) \cong \mathbb{Z} \oplus(\mathbb{Z} / 2)^{3}$ is generated by $e_{9}, e_{10}+e_{11}$ and $e_{12}+\cdots+e_{16}$ subject to the relations $2 e_{10}+2 e_{11}=0$ and $\sum_{i=12}^{17} 2 e_{i}=0$. Finally, $T(P, \mathcal{F}) \cong \mathbb{Z}^{2}$ is generated by $e_{0}, 2 e_{1}+2 e_{2}$ and $2 e_{3}+\cdots+2 e_{8}$.

\section{REFERENCES}

[1] J. Alperin, M. Broué, Local Methods in Block Theory, Ann. Math. 110 (1979), 143-157.

[2] C. Broto, R. Levi, B. Oliver, The homotopy theory of fusion systems, J. Amer. Math. Soc. 16 (2003), 779-856.

[3] M. Broué, L. Puig, A Frobenius theorem for blocks, Invent. Math. 56 (1980), 117-128.

[4] S. Bouc, Tensor induction of relative syzygies, J. reine angew. Math. 523 (2000), 113-171.

[5] S. Bouc, The Dade group of a p-group, Invent. Math., 164 (2006), 189-231.

[6] S. Bouc and N. Mazza, The Dade group of (almost) extraspecial p-groups, J. of pure and applied Algebra 192 (2004), 21-51.

[7] S. Bouc and J. Thévenaz, The group of endo-permutation modules, Invent. Math. 139 (2000), $275-349$.

[8] S. Bouc and J. Thévenaz, Gluing torsion endo-permutation modules, preprint.

[9] S. Bouc and J. Thévenaz, A sectional characterization of the Dade group, preprint.

[10] J. Carlson, N. Mazza and D. Nakano, Endotrivial modules for finite groups of Lie type, J. Reine Angew. Math., 595 (2006) 93-120.

[11] J. Carlson and J. Thévenaz, Torsion endo-trivial modules, Algebras and Representation Theory, 3 (2000), 303-335.

[12] J. Carlson and J. Thévenaz, The classification of endo-trivial modules, Invent. Math. 158 (2004), no. $2,389-411$.

[13] E. C. Dade, Endo-permutation modules over p-groups, I, II, Ann. Math. 107 (1978), 459-494, 108 (1978), 317-346.

[14] E. C. Dade, Extending endo-permutation modules, preprint (unpublished), 1982.

[15] B. Huppert, Endliche Gruppen I, Springer-Verlag, Berlin, 1967.

[16] R. Kessar, Introduction to Block Theory, in: Group Representation Theory (edts. M. Geck, D. Testerman, J. Thévenaz), EPFL Press, Lausanne (2007), 47-77

[17] M. Linckelmann, Introduction to Fusion systems, in: Group Representation Theory (edts. M. Geck, D. Testerman, J. Thévenaz), EPFL Press, Lausanne (2007), 79-113

[18] N. Mazza, Modules d'endo-permutation, Ph.D. Thesis, Lausanne, 2003.

[19] L. Puig, Structure locale dans les groupes finis, Bull.SocMath.France, Mémoire 47 (1976).

[20] L. Puig, Affirmative answer to a question of Feit, J. Algebra 131 (1990), no. 2, 513-526.

[21] L. Puig, Une correspondence de modules pour les blocs à groupes de défaut abéliens, Geom Dedicata $\mathbf{3 7}$ (1991), 9-43.

[22] A. Ruiz, A. Viruel, The classification of p-local finite groups over the extraspecial group of order $p^{3}$ and exponent p, Math. Z. 248 (2004), no. 1, 45-65.

[23] R. Stancu, Almost all generalized extraspecial p-groups are resistant, J. Algebra 249 (2002), no. 1, 120-126.

[24] J. M. Urfer, Modules d'endo-p-permutation, Ph.D. Thesis, Lausanne, 2006. 